\title{
Estrategias comunicativas para enfrentar las dificultades al hablar en inglés en una universidad pública de Colombia*
}

\author{
Communicative Strategies to Cope with the Difficulties to Speak English in a Colombian Public University \\ Estratégias comunicativas para enfrentar dificuldades para falar inglês em uma universidade pública na \\ Colômbia
}

\author{
Juan Fernando Gómez ${ }^{\mathrm{a}}$ \\ Tecnológico de Antioquia, Colombia \\ jgomez2@tdea.edu.co \\ ORCID: https://orcid.org/0000-0003-1004-6793 \\ Jorge Emiro Restrepo \\ Tecnológico de Antioquia, Colombia \\ ORCID: https://orcid.org/0000-0001-8790-7454
}

DOI: https://doi.org/10.11144/Javeriana.syp39-76.eced

Eleonora Parra Fernández

Universidad Autónoma de Chile, Chile

ORCID: https://orcid.org/0000-0001-9735-2528

Recibido: 05 Enero 2020

Aceptado: 28 Septiembre 2020

Publicado: 20 Diciembre 2020

\section{Resumen:}

Las estrategias comunicativas pueden ser utilizadas para enfrentar los problemas que surgen durante la interacción oral en el aula. El estudio plantea dos objetivos: identificar las estrategias más empleadas por estudiantes universitarios para el manejo las dificultades en la comunicación oral en inglés y describir las diferencias en la frecuencia de uso de estas estrategias entre ambos niveles y sexos. La investigación corresponde a un enfoque cuantitativo, de tipo descriptivo, con un diseño trasversal, con una población de 542 estudiantes. Las estrategias más utilizadas por esta muestra son las de intento de pensar en inglés y de reducción y alteración del mensaje. Se halló una diferencia entre ambos niveles en las estrategias orientadas hacia la adecuación. Por otro lado, los hombres hacen mayor uso de las estrategias socioafectivas, mientras que las mujeres utilizan más las de abandono del mensaje.

Palabras clave: competencia comunicativa, conciencia lingüística, estrategias comunicativas, habilidad del habla, tareas comunicativas.

\section{Abstract:}

Communicative strategies can be used to cope with the problems arising in the oral interactions in a classroom. This study sets out two objectives: To identify the most frequently used strategies by college students to cope with oral communication difficulties in English; and to describe the differences in use frequency regarding these strategies between both levels and genders. This research is conducted as a descriptive quantitative approach, cross-sectional, among a population of 542 students. Strategies most frequently used by this sample include: an attempt to think in English, and shortening and changing the meaning. A difference was found between both levels regarding the strategies intended to make messages appropriate. On the other hand, men use more frequently social-affective strategies while women use more frequently the strategy to discard the message.

Keywords: communicative competence, linguistic awareness, communicative strategies, speech capability, communicative tasks.

\section{Resumo:}

As estratégias comunicativas podem se usar para lidar com problemas surgidos durante a interação oral na sala de aula. $\mathrm{O}$ estudo levanta dois objetivos: identificar as estratégias mais usadas por discentes universitários para lidar com das dificuldades na comunicação oral em inglês e descrever as diferenças na frequência de uso dessas estratégias entre ambos os níveis e sexos. A pesquisa tem um enfoque quantitativo, descritivo, com desenho transversal, com uma população de 542 alunos. As estratégias mais utilizadas por esta amostra são as de tentativa de pensar em inglês e redução e alteração da mensagem. Achou-se diferença entre ambos os níveis nas estratégias voltadas para a adequação. Por outro lado, os homens fazem mais uso das estratégias socioafetivas, enquanto as mulheres usam mais o abandono da mensagem.

Palavras-chave: competência comunicativa, consciência linguística, estratégias comunicativas, habilidade da fala, tarefas comunicativas.

Notas de autor

$$
{ }^{a} \text { Autor de correspondencia. Correo electrónico: jgomez2@tdea.edu.co }
$$




\section{Introducción}

De acuerdo con el Ministerio de Educación Nacional de Colombia (2016), es necesario que, en este mundo globalizado, las personas desarrollen la capacidad de manejar un segundo idioma. El bilingüismo es entonces un factor fundamental para hacer frente a este fenómeno, pues proporciona habilidades lingüísticas para comunicarse eficientemente en diferentes contextos, permitiendo la apropiación de conocimientos en ciencia, telecomunicaciones y tecnología, y posibilitando la comprensión de las formas de expansión de los mercados internacionales, entre otras. En efecto, el dominio del idioma inglés se convierte en una plataforma capaz de preparar a los profesionales para competir en el mercado laboral (Chairat, 2017) y ofrecer mejores oportunidades laborales (Pandey y Pandey, 2014). Al respecto, Harmer (2010) afirma que si una persona habla inglés, debe tener la destreza de comunicar diferentes temas en diversas situaciones e intercambiar información a partir de las funciones del lenguaje.

El Ministerio de Educación de Colombia acogió el Marco Común Europeo de referencia para las lenguas (MCERL) como el estándar internacional que determina el nivel de competencia óptimo en un idioma y el cual es utilizado en todo el mundo para la evaluación, enseñanza y aprendizaje de diversos idiomas (Consejo de Europa, 2002). En este marco, se especifica cómo las estrategias comunicativas son activadas por los estudiantes cuando llevan a cabo actividades que implican la interacción oral, y asumen los roles de emisor y receptor, logrando a través de estas compensar las posibles insuficiencias lingüísticas en la lengua meta, suscitadas en el acto comunicativo. Para Nakatani (2010), las estrategias comunicativas se refieren a mecanismos usados por los estudiantes para resolver las dificultades que se producen durante la interacción y de este modo, facilitan la obtención de la competencia comunicativa.

Actualmente, el Área de Idiomas del Tecnológico de Antioquia, institución universitaria, tiene una propuesta de enseñanza del inglés a través de dos tipos de estrategias: la primera promueve el desarrollo de la competencia comunicativa por medio del enfoque pedagógico Aula invertida (Flipped Classroom), propuesto por Bergmann y Sams (2012), en la cual se trasfiere el desarrollo de ciertos procesos de aprendizaje fuera del aula por parte de los estudiantes; por tanto, en cada encuentro el docente optimiza el tiempo para generar experiencias de aprendizaje y prácticas dirigidas a la generación de conocimiento. La segunda corresponde a Task Based Learning o TBL, cuya traducción al español es Aprendizaje basado en tareas o ABP. Moore (2018) la define como un enfoque en el cual se asigna una tarea como una unidad de análisis que permite practicar la lengua de una manera comunicativa.

No obstante, estas prácticas de enseñanza no siempre resultan efectivas, en razón de que una gran cantidad de estudiantes pertenecientes a los niveles de dominio lingüístico A2 y B1 desconocen estrategias que les permitan superar las deficiencias lingüísticas que se presentan durante la realización de tareas que implican la comunicación oral en el aula. De igual modo, los estudiantes evidencian dificultades para encontrar las palabras, expresiones y estructuras gramaticales requeridas para transmitir el mensaje de manera adecuada y con una intensión comunicativa. Los problemas anteriormente mencionadas son consistentes con diversos estudios empíricos sobre la habilidad del habla en una lengua extranjera (Keim y Delgar, 2019; Shirkhani et al., 2020)

Conforme al escenario descrito anteriormente, los objetivos del estudio se enfocaron en identificar las estrategias comunicativas más empleadas por estudiantes universitarios para el manejo de las dificultades en la comunicación oral en inglés, para lo cual resulta de interés describir las diferencias en la frecuencia de uso de las estrategias comunicativas entre ambos niveles y sexos: sexo, como distinción biológica hombre y mujer, y los niveles de dominio de la lengua que han sido establecidos por el Marco Común Europeo de Referencia para las Lenguas (MCERL). 


\section{Fundamentación teórica}

\section{Marco Común Europeo de Referencia para las Lenguas (MCERL)}

El MCERL es el estándar internacional empleado para establecer el nivel de competencia sobre un idioma y es el instrumento empleado en todo el mundo para la evaluación, enseñanza y aprendizaje de diversos idiomas (Consejo de Europa, 2002). Dicho marco ha delimitado las capacidades que debería desarrollar las personas en función de cada nivel (A1, A2, B1, B2, C1 y C2) en cinco dimensiones. Estas dimensiones presentan de manera unificada los criterios a ser considerados para cada nivel del aprendizaje de un idioma: Comprensión de lectura, Interacción oral, Expresión oral y Expresión escrita. En este estándar europeo se hace mención a la importancia de la realización de tareas comunicativas, puesto que esto exige poner en práctica el uso de estrategias para la comunicación.

La aplicación de la competencia comunicativa y los diferentes métodos para generarla se han convertido en un área de interés para la investigación sobre la enseñanza de una lengua extranjera (Salazar-Obeso, 2015). Además, esta se constituye en el tema central presentado por el Ministerio de Educación Nacional de Colombia en el Programa Formadores Nativos Extranjeros (Ministerio de Educación Nacional, 2016), con el propósito de fortalecer la propuesta de bilingüismo en el país. De acuerdo con Dell Hymes (1971 citado por Castillo, 2013), la competencia comunicativa está ligada a ciertos aspectos: saber en qué momento hablar, saber cuándo no es indicado hacerlo, saber sobre qué hablar, con quién llevar acabo el acto comunicativo y seleccionar el lugar y la manera en que debe realizarse. Al respecto, Ahmed y Pawar (2018) conciben esta competencia como la habilidad de comunicarse, lo cual implica la capacidad para hablar, escribir, tener el conocimiento de la lengua y la destreza para usar el lenguaje en situaciones reales para satisfacer diversas necesidades comunicativas.

Cuando se hace referencia a la comunicación oral en el contexto de enseñanza y aprendizaje de un segundo idioma, para este caso el inglés, es necesario pensar en el uso de estrategias que ayuden a los estudiantes a comunicarse eficientemente pese a sus posibles limitaciones lingüísticas. Al respecto, Ounis (2016) afirma que se existe un consenso en la comunidad académica que respalda la idea de que las estrategias comunicativas ayudan a superar las deficiencias sobre el conocimiento de la lengua meta, y permite que los estudiantes puedan mantener una conversación.

De acuerdo con Nakatani (2006), estas estrategias se constituyen en comportamientos estratégicos que los aprendices de una segunda lengua emplean en el momento de enfrentar las dificultades que surgen durante el acto comunicativo. Dichos comportamientos se evidencian en las acciones ejecutadas por algunos estudiantes en el momento de realizar tareas comunicativas que implican la expresión oral, con el propósito de no abandonar su intento por comunicarse en la lengua meta, y así poder superar de algún modo las posibles insuficiencias que surgen durante este acto.

\section{Inventario de Estrategias de Comunicación Oral (OCSI) y estudios relacionados con la habilidad del habla}

El instrumento especializado OCSI fue creado por Nakatani (2006) y contempla dos cuestionarios que contienen las estrategias empleadas para afrontar las dificultades al hablar y para enfrentar los problemas de escucha durante la ejecución de tareas comunicativas. La sigla OCSI en español remite al Inventario de Estrategias de Comunicación Oral, que ha sido ampliamente utilizado en diversos contextos y con poblaciones de estudiantes universitarios pertenecientes a instituciones públicas y privadas de varios países: Túnez (Ounis, 2016), Tailandia (Chairat, 2017), Vietnam (Tuyen et al., 2020), entre otros. 
El cuestionario que contiene las estrategias para enfrentar dificultades al hablar está conformado por los siguientes factores: 1 ) estrategias socioafectivas (reducen la ansiedad, el temor a equivocarse e incentivan la confianza mutua; 2) estrategias orientadas hacia la fluidez (fortalecen la capacidad para expresar las ideas con facilidad y espontáneamente); 3) de negociación del significado (confirman si el receptor ha comprendido e implican la clarificación, repetición y confirmación del mensaje); 4) orientadas hacia la exactitud (uso adecuado de la gramática al expresarse); 5) de reducción y alteración del mensaje (reducción del mensaje original o empleo de expresiones similares para lograr la comprensión; 6) no verbales (facilitan la comprensión a través del contacto visual y expresiones faciales); 7) de abandono del mensaje (manera de superar la barrera por no saber cómo continuar el mensaje) y 8) de intento de pensar en inglés (la traducción del español al inglés). El segundo cuestionario está dirigido hacia las estrategias para enfrentar dificultades al escuchar y está constituido por seis factores.

Ounis (2016) empleó el instrumento OCSI para examinar las rupturas en la comunicación oral con un grupo de 100 estudiantes del Instituto Superior de Idiomas de Gabés, en Túnez. Los resultados revelaron cómo las estrategias correspondientes a la negociación del significado, no verbales, de reducción del mensaje y de alteración son las que se emplean con mayor frecuencia. Entre los hallazgos, se encuentran que las estrategias de negociación de significado, socioafectivas y orientadas hacia el manejo de los problemas al hablar están asociados a los estudiantes con un nivel de dominio alto del inglés. Se concluyó que los estudiantes con un bajo nivel de dominio deberían familiarizarse y ser conscientes de la importancia de las estrategias comunicativas a través de la instrucción formal en el aula.

Por su parte, Chairat (2017) se propuso identificar las estrategias comunicativas empleadas por 67 estudiantes de último año en especialización en inglés, en una universidad pública de Tailandia a partir del instrumento OCSI. Los resultados evidenciaron que las estrategias no verbales y las de reducción del mensaje fueron las más usadas para afrontar las dificultades que se presentaron en el desarrollo de las actividades comunicativas. Además, la estrategia de intentar pensar en inglés fue la que menor porcentaje reportó. En general, las estrategias que más utilizaron los encuestados correspondieron a las no verbales, reducción de mensaje y alteración y negociación del significado. El estudio definió que es importante que los docentes de lengua extranjera incluyan en el currículo el entrenamiento en estrategias comunicativas y los incentivos para concientizar a los estudiantes para que las utilicen.

El OCSI fue empleado por Jindathai (2017) para identificar las estrategias en el desarrollo de la comunicación oral utilizadas por 361 estudiantes universitarios del Instituto de Tecnología Thai-Nichi y de la Universidad de Mahanakorn, y para hallar las posibles diferencias con relación al sexo y la autopercepción que se tiene sobre su habilidad para expresarse de forma oral. Los resultados indicaron cómo las estrategias más empleadas son las de reducción del mensaje y de alteración, y las usadas con menos frecuencia las orientadas a la adecuación. No se reportaron discrepancias significativas entre las dificultades presentadas en ambos sexos en relación con los factores que conforman los cuestionarios.

En el contexto de Vietnam, Tuyen et al. (2020) investigaron las estrategias utilizadas para enfrentar las dificultades en la producción oral a través del OCSI con un grupo de 213 estudiantes de una universidad pública. Los resultados indicaron que las estrategias orientadas a la fluidez, reducción y alteración del mensaje fueron utilizadas con mayor frecuencia. Las de menor frecuencia correspondieron a las dirigidas hacia la exactitud. El estudio no reportó diferencias en el empleo de las estrategias para enfrentar las dificultades en la comunicación oral entre los tres niveles de dominio estimados. A manera de conclusión, los autores proponen la enseñanza implícita o explícita de las estrategias comunicativas al igual que la creación de ambientes que promuevan su uso y de este modo puedan suplir los vacíos lingüísticos. 


\section{Método}

\section{Diseño de la investigación}

El estudio plantea dos objetivos: identificar las estrategias más empleadas por estudiantes universitarios para el manejo de las dificultades en la comunicación oral en inglés y describir las diferencias en la frecuencia de uso de estas estrategias entre los niveles A2 y B1 y el sexo. Para tal propósito, la metodología de esta investigación correspondió a un enfoque cuantitativo, de tipo descriptivo, con un diseño trasversal y un muestreo no probabilístico por conveniencia.

\section{Contexto y participantes}

Los participantes de esta investigación están constituidos por 542 estudiantes ( 380 mujeres y 162 hombres), los cuales se dividieron en dos estratos poblacionales de acuerdo al nivel de dominio de la lengua: cursantes de la asignatura de inglés 3 y 4 (346 estudiantes en nivel A2) e inglés 5 y 6 (196 estudiantes en nivel B1). La población encuestada se encuentra realizando carreras tecnológicas en diversos programas académicos en el Tecnológico de Antioquia-Institución Universitaria, ubicado en la ciudad de Medellín, Colombia. Los niveles de dominio lingüístico se determinaron por la institución al inicio del semestre académico en conformidad con las horas de exposición al idioma en los diversos cursos y criterios definidos por el MCERL. La selección de la muestra del estudio se realizó de manera aleatoria a partir de los grupos que se conformaron desde la oficina de admisiones y registro.

Todos los participantes se encuentran cursando la asignatura de inglés con una intensidad de cuatro horas semanales, bajo dos enfoques de aprendizaje: la primera es Flipped classroom (Aula invertida) y la segunda corresponde a Aprendizaje Basado en Tareas (Task Based Learning). El promedio de edad de los encuestados correspondió a 22.8 años. Se aclara que en este estudio solo se abordaron las estrategias para enfrentar las dificultades suscitadas al momento de hablar durante la realización de tareas comunicativas, las cuales fueron propuestas por los docentes, así: juegos de roles, entrevistas, reporte de videos, narración de historias, discusión de temas controvertidos en parejas y equipos, entre otras.

\section{Instrumento de recolección de datos}

Los datos obtenidos provienen de la aplicación del instrumento especializado denominado OCSI, creado por Nakatani (2006). Se empleó la versión traducida al español por Bonilla-Traña (2016), la cual fue cotejada por lingüistas expertos. El cuestionario usado corresponde a las estrategias para enfrentar dificultades que surgen al hablar durante el desarrollo de tareas comunicativas, constituido por 32 enunciados distribuidos en ocho factores de la siguiente manera: 1) 6 ítems; 2) 6 ítems; 3) 4 ítems; 4) 5 ítems; 5) 3 ítems; 6) 2 ítems; 7) 4 ítems; 8) 2 ítems (Tabla 1). Para indicar la frecuencia de uso de la estrategia comunicativa en cada ítem, se utilizó la escala Likert de la siguiente forma: 1 (Nunca), 2 (Rara vez), 3 (A veces), 4 (Normalmente) y 5 (Siempre). De acuerdo con Yaman y Özcan (2015), los criterios para evaluar el nivel de frecuencia del uso de las estrategias comunicativas comprenden los siguientes valores: frecuencia de uso baja $(1-2,49)$, frecuencia de uso media $(2,5-3,49)$ y frecuencia de uso alta $(3,5-5)$. El cuestionario fue diligenciado por los participantes durante un solo contacto en el semestre académico y se empleó la estadística descriptiva para analizar los datos provenientes del instrumento, el cual corresponde a las estrategias para enfrentar las dificultades en la producción oral. Los análisis estadísticos se realizaron con el programa IBM SPSS. v. 24. 
Se empleó el test de alfa de Cronbach para determinar el nivel de confiabilidad de la consistencia interna de los ítems que conforman el cuestionario de expresión oral. El coeficiente obtenido para esta muestra fue 0,865, lo que indicó que este cuestionario tiene una alta consistencia por la cercanía al valor superior de alfa, el cual es 1. El test de Kolmogorov-Smirnov confirmó que los datos no siguen una distribución normal en ninguna de las variables de ambos cuestionarios.

Para determinar las diferencias sobre la frecuencia de uso de las estrategias entre ambos niveles y sexos se aplicó la prueba no paramétrica $U$ de Mann-Whitney, pues se trata de dos muestras independientes que no cumplen el supuesto de normalidad. El nivel de significancia de alfa $(\alpha)$ establecido fue del 0,05 (5\%) y esto significa que un $p$-valor por debajo del alfa determinado indica rechazo a la hipótesis nula y se acepta la alterna. La hipótesis alterna planteada en relación con los niveles fue: se presentan diferencias sobre la frecuencia de uso de las estrategias comunicativas entre los niveles A2 y B1; en cuanto al sexo, se estableció: existen diferencias sobre la frecuencia de uso de las estrategias comunicativas entre los hombres y las mujeres.

\section{Resultados y discusión}

\section{Estrategias comunicativas empleadas por toda la población}

Los resultados revelaron la existencia de cinco factores que se encuentran en un rango de frecuencia alta, mientras los restantes se ubicaron en la media (Tabla 1). El de Intento de pensar en inglés obtuvo la frecuencia de uso más alta, con una $M=3,84$. En segundo lugar, el de Reducción y alteración del mensaje indicaron un valor $M=3,77$. En el tercero, las Estrategias no verbales presentó una $M=3,66$. En el cuarto, el factor correspondiente a las estrategias Orientadas hacia la fluidez mostró una $M=3,6$. A pesar de indicar una frecuencia media de uso, las estrategias que menos emplean estos estudiantes para la realización de tareas comunicativas correspondieron al factor de las Orientadas hacia la adecuación, ya que fue una $M=3,35$, y en el último lugar, las de Abandono del mensaje, con una $M=3,13$.

TABLA 1

Estadisticos descriptivos de cada factor

\begin{tabular}{lcccc}
\hline \multicolumn{1}{c}{ Factores } & Media & $\begin{array}{c}\text { Desviación } \\
\text { Estándar }\end{array}$ & Posición* & $\begin{array}{c}\text { Frecuencia } \\
\text { de uso }\end{array}$ \\
\hline Factor 1. Socioafectivas & 3,41 & 0,66 & 6 & Media \\
\hline Factor 2. Orientadas hacia la fluidez & 3,6 & 0,72 & 4 & Alta \\
\hline Factor 3. De negociación del significado & 3,59 & 0,82 & 5 & Alta \\
\hline Factor 4. Orientadas hacia la adecuación & 3,35 & 0,79 & 7 & Media \\
\hline $\begin{array}{l}\text { Factor 5. De reducción y alteración del } \\
\text { mensaje }\end{array}$ & 3,77 & 0,7 & 2 & Alta \\
\hline Factor 6. Estrategias no verbales & 3,66 & 0,87 & 3 & Alta \\
\hline Factor 7. De abandono del mensaje & 3,13 & 0,72 & 8 & Media \\
\hline Factor 8. De intento de pensar en inglés & 3,84 & 0,88 & 1 & Alta \\
\hline
\end{tabular}

Nota. *De 1 a 6 hace referencia al orden en el uso de la estrategia de acuerdo con la media.

Fuente: elaboración propia.

Las estrategias empleadas por esta población con mayor frecuencia en el momento de compensar las deficiencias lingüísticas que surgen durante el desarrollo de tareas que involucran la comunicación oral, corresponden al Factor 8 De intento de pensar en inglés. En este factor, un 91,3\% de los encuestados primero piensan en una frase conocida en inglés y después intentan cambiarla adecuándola a la situación y el 28,8 $\%$ piensan en inglés en el momento de realizar tareas que implican la comunicación oral. De acuerdo con Nakatani (2006), estas estrategias son útiles para los estudiantes que piensan en la segunda lengua en el momento de realizar su discurso, y al parecer, los estudiantes inmersos en los porcentajes anteriormente expuestos recurren a estas. En efecto, una persona, al emplear estas estrategias, tiende a pensar en inglés y evitar pensar en la lengua nativa, que para este caso, es el español. Este resultado llama la atención ya que esta muestra se encuentra en un contexto donde no se emplea dicha lengua extranjera para propósitos comunicativos en 
ambientes de interacción cotidianos, y es exclusivamente utilizada durante los cursos de inglés, donde se llevan a cabo tareas en el aula para desarrollar la competencia comunicativa.

Pensar en inglés implica tener un grado de conciencia lingüística en el que se origina la habilidad para reflexionar sobre la naturaleza y las funciones del lenguaje. En lo que atañe a la conciencia lingüística, De Villa (2018) sostiene que esta le confiere al estudiante los recursos suficientes para solucionar cognitivamente un problema comunicativo en cualquier contexto. Aún más, Gombert (1990 citado por Signoret, 2003), argumenta que dicha conciencia es aplicada a los niveles lingüísticos de la lengua (fonológico, semántico, lexical, sintáctico, morfosintáctico) y de igual forma al uso textual y pragmático. Al respecto, Djandue (2012) explica que se aprende a hablar en lugar de aprender a pensar, y la habilidad de pensar es un resultado lógico del saber hablar, y hablar en una lengua determinada. De igual modo, no existe un pensamiento sin palabra, pues pensar indica hablar y el hablar indica un ejercicio de pensamiento. Esta declaración conlleva a suponer que el ejercicio de pensamiento se traduce en un intercambio de ideas con otras personas en forma oral e, inclusive, en la realización de monólogos a nivel mental o en voz alta. De igual modo, esto indica cómo se presenta una interrelación entre el lenguaje y el pensamiento, por lo cual no sería posible conocer los pensamientos sin recurrir al lenguaje, y es este el mecanismo que hace posible exponer los pensamientos.

Ante la situación planteada, podría decirse que las estrategias del Factor 8 han sido probablemente las más empleadas $(M=3,84)$, debido a su disposición por comunicarse en inglés (Willingness to Communicate o WTC). Wood (2016) se refiere a la WTC como a la disposición que un individuo tiene para comunicarse, lo cual se constituye en un estado mental que hace que se incline a participar en una conversación en una situación determinada debido a su estado de autoconfianza.

En cuanto a la WTC, Bergil (2016) explica que la competencia lingüística en sí misma no causa restricciones para hablar, del mismo modo que aquellos con limitaciones en este aspecto pueden hablar continuamente. Esto permite considerar que posiblemente un estudiante ubicado en un nivel más alto, no necesariamente ha alcanzado la competencia lingüística requerida, pero quizás, como afirma Farzam (2017), su disposición para comunicarse en una lengua extranjera se incrementa a través del conocimiento y empleo de las estrategias comunicativas.

En contraste, las estrategias que ocuparon el último lugar fueron las de abandono del mensaje $(M=3,13$ frecuencia media). Este factor contiene las estrategias empleadas por el interlocutor cuando encuentra algún problema y decide detenerse y dejar el mensaje inconcluso, o simplemente carece de los medios lingüísticos que le permitan dar una respuesta. En relación con este factor, un $27,7 \%$ deja un mensaje inacabado a causa de alguna dificultad; un 70,7 \% piden ayuda al no saber comunicarse; un 23,2 \% desisten al no poder hacerse entender y un $28,8 \%$ deciden no hablar debido a su incapacidad, y solo dicen algunas palabras. Al respecto, podría afirmarse que estas cifras no solo dan cuenta de las insuficiencias lingüísticas, sino también que aluden a ciertos factores que tienen lugar en el aprendizaje de una lengua extranjera y varían en cada individuo: aspectos cognitivos, afectivos, motivacionales, aptitudinales, actitudinales, de personalidad y conciencia de estar adquiriendo otra lengua.

Las estrategias correspondientes al factor de reducción y alteración del mensaje ocuparon el segundo lugar en frecuencia de uso $(\mathrm{M}=3,77)$. Los resultados revelaron que un $86,9 \%$ reducen el mensaje y usan expresiones simples; un $97,8 \%$ usan palabras familiares; un $86,3 \%$ reemplaza el mensaje original. Estos resultados indican que un alto porcentaje de esta muestra recurre a dichas estrategias con la intensión de simplificar lo que desea decir, mediante el uso de otras palabras o expresiones conocidas y a través de la dicción de enunciados simples (o simplemente se deja a un lado dicha idea debido a la incapacidad de producir el mensaje original). En esta instancia, tienen lugar aspectos como cambio de código (code switching), transferencia e interlenguaje. Por ejemplo, un estudiante podría decir big hole, en lugar de emplear la oración It was a huge crater. En efecto, quienes poseen un dominio mayor de la lengua, tienen un repertorio semántico más amplio y un uso de la sintaxis y la gramática más complejo. Se considera, entonces, que el uso frecuente de las estrategias de reducción y de abandono del mensaje indica que las tareas comunicativas realizadas generan dificultades en 
los estudiantes, y esto hace creer que no se presenta un claro dominio de los patrones lingüísticos de la lengua meta.

Las estrategias no verbales obtuvieron el tercer lugar $(\mathrm{M}=3,66)$, lo que las ubica en un rango alto de uso. Un $86,2 \%$ usa la estrategia de establecer contacto visual al hablar, y un $86,7 \%$ acude a los gestos y expresiones faciales si no logra encontrar las palabras adecuadas. Este porcentaje refleja que, muy probablemente, los estudiantes utilizan el lenguaje corporal y prestan atención a las reacciones de los interlocutores para comprender el mensaje. Estos son recursos de ayuda para evitar las interrupciones constantes, logrando fluidez en el discurso.

\section{Diferencias frecuencia de uso de las estrategias comunicativas entre ambos niveles}

En lo concerniente a los niveles A2 y B1, no se presentaron diferencias significativas en la frecuencia de uso de las estrategias entre ambos grupos, en ninguno de los factores (Tabla 2), lo cual fue demostrado con la prueba . de Mann-Whitney.

Esto podría explicarse debido a que los individuos de este nivel poseen una mayor conciencia del idioma (language awareness), la cual, según García (2009), se refiere al conocimiento del idioma o a la capacidad de usarlo de manera adecuada en diferentes situaciones y al conocimiento de la lengua que implica los sistemas de gramática, fonología y vocabulario. La exposición al idioma de una persona que posee un nivel lingüístico B1 es superior a la de una en A2 y, por lo tanto, aquella tendrá una mayor conciencia del uso de la lengua. Evidentemente, es más factible que quienes tienen este nivel tiendan a usar en mayor proporción estrategias de negociación de significado para controlar problemas de ansiedad y de desconocimiento de cierto vocabulario, en comparación con los de un nivel más bajo, para el caso A2. Estos últimos acuden en mayor proporción a evitar los problemas comunicativos con estrategias de reducción, alteración y abandono del mensaje debido a su incapacidad lingüística, lo que es constatado por Jindathai (2017).

Según el Consejo de Europa (2002), el nivel A2 corresponde a los usuarios básicos de la lengua que conocen pocas palabras y emplean fórmulas memorizadas al momento de hablar. En contraste, los usuarios independientes $\mathrm{B} 1$ son quienes poseen un vocabulario más amplio para expresarse aunque dubitativamente, apelan a los circunloquios y requieren de una planificación gramatical, léxica y de una corrección. La descripción anterior deja ver claramente la existencia de varios aspectos que comprenden la competencia comunicativa, que es fundamental para dar respuesta a las necesidades que un individuo pueda tener para comunicarse en un contexto determinado (Berenguer-Román et al., 2016).

$\mathrm{Al}$ respecto, la revisión de la literatura realizada por Al Alawi (2016) señala cuatro componentes de este conjunto de destrezas: competencia gramatical, discursiva, sociolingüística y estratégica. En otros términos, las dos primeras se refieren a los dispositivos del sistema lingüístico, y los restantes comprenden los elementos funcionales de la comunicación. Causa sorpresa encontrar que, en ambos niveles de dominio de la lengua, los valores se ubican en los mismos rangos en todos los factores, considerando los reportes de estudios donde el mayor uso de las estrategias comunicativas es de aquellos con un nivel de dominio del lenguaje más alto (Metcalfe \& Noom-Ura, 2013; Ounis, 2016; Jindathai, 2017). La literatura revisada en esta investigación confirma que las estrategias para la expresión oral utilizadas por cada individuo varían según el nivel de dominio en que se encuentre.

Con los resultados presentados es difícil establecer la manera como se han expandido los recursos comunicativos usados por los estudiantes cuando están en un nivel más alto, pues no se encuentran diferencias estadísticamente significativas y todas las frecuencias de uso de las estrategias en ambos niveles se ubican en los mismos rangos en cada factor (Tabla 2). Ciertamente, es más probable que aquellos con un nivel B1 tiendan a usar en mayor proporción estrategias de negociación de significado para controlar posibles problemas de ansiedad y de desconocimiento de cierto vocabulario, en comparación con los de un nivel más bajo, quienes 
tenderían a evitar los problemas comunicativos con estrategias de reducción y abandono del mensaje, como lo confirmó Al Alawi (2016).

Adicionalmente, los de mayor nivel no tendrían problemas en emplear la circunlocución puesto que tienen un mayor conocimiento de la lengua, mientras los del nivel A2 tendrían un estado de ansiedad mayor debido a su carencia lexical, y esto generará una incapacidad para tener una conversación fluida y, por tal motivo, desistirían del intento comunicativo debido a su incapacidad lingüística. De acuerdo con Ounis (2016), aquellos con un nivel bajo usualmente recurren a la lengua materna con mayor frecuencia que los de un nivel de dominio más alto. De aquí se presume que tienen mayores necesidades de suplir los vacíos lingüísticos en el momento de desarrollar tareas orales.

TABLA 2

Diferencias entre ambos niveles en la frecuencia de uso de las estrategias para hablar

\begin{tabular}{lcccccc}
\hline \multicolumn{1}{c}{ Factores } & \multicolumn{6}{c}{$\mathbf{A 2}$} \\
\hline & $\boldsymbol{M}$ & $\boldsymbol{D E}$ & $\boldsymbol{M}$ & $\boldsymbol{D E}$ & $U$ & $\boldsymbol{p}^{*}$ \\
\hline Factor 1. Socioafectivas & 3,39 & 0,64 & 3,46 & 0,7 & 31443,5 & 0,158 \\
\hline Factor 2. Orientadas hacia la fluidez & 3,59 & 0,71 & 3,61 & 0,72 & 33674,5 & 0,894 \\
\hline Factor 3. De negociación del significado & 3,58 & 0,83 & 3,6 & 0,78 & 33826,5 & 0,963 \\
\hline Factor 4. Orientadas hacia la adecuación & 3,32 & 0,8 & 3,42 & 0,78 & 31395,5 & 0,15 \\
\hline $\begin{array}{l}\text { Factor 5. De reducción y alteración del } \\
\text { mensaje }\end{array}$ & 3,74 & 0,72 & 3,81 & 0,67 & 32417,5 & 0,389 \\
\hline Factor 6. Estrategias no verbales & 3,65 & 0,84 & 3,68 & 0,92 & 33057,5 & 0,622 \\
\hline Factor 7. De abandono del mensaje & 3,12 & 0,72 & 3,15 & 0,73 & 32974 & 0,592 \\
\hline Factor 8. De intento de pensar en inglés & 3,82 & 0,87 & 3,88 & 0,89 & 32625 & 0,45 \\
\hline
\end{tabular}

*Significativa para $\mathrm{p}<0.05$.

Fuente: elaboración propia.

La explicación para el hecho de no encontrar diferencias significativas entre ambos niveles en esta muestra se atribuye probablemente a que algunos docentes aún no se han apropiado del modelo de enseñanza Flipped classroom y Task Based Learning o TBL. Estos enfoques involucran las tecnologías de la información para que el estudiante tenga procesos de aprendizaje autónomos y genere experiencias de comunicación reales capaces de promover el desarrollo de las habilidades comunicativas. Además, estos dan la oportunidad a los estudiantes de usar el idioma a través de tareas que hacen referencia a situaciones cotidianas. Esto hace pensar que un adecuado desarrollo de los enfoques de enseñanza anteriormente mencionados, permite establecer una distinción entre las competencias que debe tener un estudiante para hacer la transición del nivel A2 al B1.

Se parte de la idea de que la naturaleza de la tarea y el nivel de dominio son determinantes al momento de utilizar las estrategias comunicativas (Mei y Nathalang, 2010). En efecto, un estudiante de un nivel bajo acudirá a las estrategias de reducción y alteración del mensaje (parafraseo, generalización, préstamo lingüístico, word coinage, aproximación) y a las estrategias de abandono del mensaje, pues su nivel de interlingua se encuentra en un estadio temprano. Por el contrario, en un nivel más alto, un individuo recurriría a las estrategias de negociación de significado y prestará mayor atención a las orientadas a la fluidez, lo cual es consistente con los hallazgos de Jindathai (2017).

\section{Diferencias en las frecuencia de uso de las estrategias comunicativas entre ambos sexos}

En cuanto a la diferencia del empleo de las estrategias comunicativas entre ambos sexos, la prueba $U$ de MannWhitney demostró diferencias en el Factor 1 (Tabla 3). Esto significa que, en esta muestra, los hombres hacen mayor uso de las estrategias socioafectivas, mientras las mujeres utilizan más las de abandono del mensaje para enfrentar las dificultades al hablar. Las cifras obtenidas en las medias conducen a suponer que el sexo no es un factor determinante en esta población, en cuanto a la aplicación de estrategias comunicativas ya que prácticamente las utilizan en equivalencias similares. Estas cifras permiten deducir que los hombres se preocuparon más por regular sus emociones mientras se comunicaban de manera oral y se inclinaron por expresar sus ideas sin importar los errores que pudieran cometer. En contraste, las mujeres tendieron en mayor 
medida a dejar sus respuestas inconclusas, a solicitar ayuda o a desistir de su intención debido a la incapacidad para comunicarse.

$\mathrm{Al}$ respecto, Ning et al. (2010) demostraron que ambos sexos dominan diferentes estilos de comunicación, donde el lenguaje usado por los hombres es diferente al de las mujeres, en relación con la manera en que se comportan durante los actos comunicativos. Dentro de la misma línea, Yusparizal et al. (2018) encontraron diferencias en el empleo de las estrategias entre ambos sexos en el momento de suplir los vacíos lingüísticos en diversas tareas que implican la comunicación oral. En contraste, Jindathai (2017) y Demir et al. (2018) no reportaron diferencias entre las estrategias comunicativas empleadas por los hombres y las mujeres. Al no encontrar un consenso en la literatura, los resultados obtenidos respecto a esta variable podrían atribuirse a diferencias individuales, conocimientos previos del idioma, experiencias personales, creencias sobre el aprendizaje, aspectos metodológicos y el contexto.

TABLA 3

Diferencias sobre la frecuencia de uso de las estrategias para hablar entre ambos sexos

\begin{tabular}{lcccccc}
\hline \multicolumn{1}{c}{ Factores } & \multicolumn{1}{c}{ Hombres } & \multicolumn{2}{c}{ Mujeres } & \\
\hline & $\boldsymbol{M}$ & $\boldsymbol{D E}$ & $\boldsymbol{M}$ & $\boldsymbol{D E}$ & $\boldsymbol{U}$ & $\boldsymbol{p}^{*}$ \\
\hline Factor 1. Socioafectivas & 3,53 & 0,65 & 3,37 & 0,67 & 26968 & 0,022 \\
\hline Factor 2. Orientadas hacia la fluidez & 3,66 & 0,67 & 3,58 & 0,73 & 29170,5 & 0,333 \\
\hline Factor 3. De negociación del significado & 3,67 & 0,82 & 3,55 & 0,81 & 27768 & 0,07 \\
\hline Factor 4. Orientadas hacia la adecuación & 3,43 & 0,83 & 3,32 & 0,78 & 27588 & 0,055 \\
\hline $\begin{array}{l}\text { Factor 5. De reducción y alteración del } \\
\text { mensaje }\end{array}$ & 3,82 & 0,62 & 3,75 & 0,74 & 30106,5 & 0,683 \\
\hline Factor 6. Estrategias no verbales & 3,67 & 0,86 & 3,65 & 0,87 & 30468 & 0,849 \\
\hline Factor 7. De abandono del mensaje & 3 & 0,71 & 3,19 & 0,73 & 26362 & 0,008 \\
\hline Factor 8. De intento de pensar en español & 3,78 & 0,9 & 3,87 & 0,87 & 29054 & 0,286 \\
\hline
\end{tabular}

*Significativa para $\mathrm{p}<0.05$

Fuente: elaboración propia.

\section{Conclusiones}

La presente investigación tuvo como propósito identificar las estrategias más empleadas por estudiantes universitarios para el manejo de las dificultades en la comunicación oral en inglés, así como describir las diferencias en la frecuencia de uso de estas estrategias entre ambos niveles y sexos. Los resultados evidenciaron que las estrategias más empleadas por toda la muestra corresponden a las de intento de pensar en inglés, seguido de las de reducción y alteración del mensaje. Ambos factores se encuentran ubicadas en un rango alto de uso. Por su parte, el factor de menor frecuencia correspondió al de abandono del mensaje, en un rango medio. En relación con los niveles A2 y B1, la prueba $U$ de Mann-Whitney mostró la inexistencia de diferencia en las medias de los ocho factores que componen el inventario de estrategias para enfrentar las dificultades al hablar. En cuanto al sexo, la prueba reveló que los hombres emplean las estrategias socioafectivas con mayor frecuencia, mientras las mujeres usan las de abandono del mensaje para enfrentar las dificultades al hablar.

Estos hallazgos indican que, sin importar el nivel de dominio de la lengua o el sexo, los estudiantes recurren a diversas estrategias comunicativas para hacer frente a sus limitaciones lingüísticas y, de acuerdo con Keim y Delgar (2019), son activadas cuando el interlocutor o el emisor detectan un problema en la comunicación. Se destaca que los estudiantes emplean diferentes estrategias para encontrar posibles alternativas para afrontar las rupturas en la comunicación y lograr el propósito comunicativo, lo cual es afín con los hallazgos de Tuyen et al. (2020). Esto permite pensar que existen aspectos individuales que afectan directamente la selección de estrategias, tales como: conocimientos previos, motivación, aspectos cognitivos, aptitudes, actitudes, creencias sobre el aprendizaje de la lengua, personalidad, factores afectivos, entre otros.

El habla es una habilidad de gran importancia, dado que implica la expresión de pensamientos a través de la palabra hablada con un propósito comunicativo y requiere conocimiento, claridad y fluidez. Cuando se presentan dificultades con algunos de estos aspectos durante el desarrollo de una tarea comunicativa, los interlocutores acuden a las estrategias de expresión oral para suplir las carencias lingüísticas a nivel fonológico, semántico, lexical, sintáctico, morfosintáctico. De acuerdo con Tuyen et al. (2020), los estudiantes alcanzan 
un mayor nivel de dominio de la lengua y mejoran la habilidad en la comunicación oral a partir del uso de las estrategias comunicativas y para ello se considera importante que tengan la posibilidad de afrontar diferentes retos lingüísticos. Además, Keim y Delgar (2019) sostienen que los retos cognitivos asociados con tareas comunicativas también influyen sobre a selección de estrategias.

En correspondencia con las implicaciones pedagógicas derivadas de este estudio, se sugiere que los docentes de lengua extranjera creen ambientes de clase donde se favorezca el desarrollo de la competencia comunicativa, a través de experiencias que motiven a los estudiantes a usar el inglés en forma real. Esto indica entonces el empleo de materiales auténticos que permitan a los estudiantes vivenciar el lenguaje con un propósito comunicativo, en donde las cotidianidades tengan lugar en el aula de clase: hacer una compra en almacén y regatear, registrase en un hotel, expresar acuerdo y desacuerdo, entre otras. En este sentido, ellos podrán pasar de una etapa elemental del aprendizaje a un nivel de dominio de la lengua superior, en concordancia con los descriptores del MCERL.

Se considera importante que los docentes generen y propicien espacios de reflexión para ayudar a los estudiantes a ser conscientes de la manera adecuada de aplicar las estrategias comunicativas de acuerdo a la situación. Así, recurrirán con menor frecuencia a las estrategias de abandono del mensaje, las traducciones literales y/o reducir o cambiar el mensaje debido a la incapacidad lingüística. Por lo tanto, emplearán en mayor medida las estrategias de negociación de significado y no serán tan trascendentales las estructuras y las reglas sociolingüísticas para afrontar una dificultad que surge al hablar con un usuario de la lengua inglesa. Teniendo en cuenta lo anterior, se tiene la convicción de que se reducirá la ansiedad, se incrementará la seguridad y se generarán actitudes más positivas. durante el desarrollo de tareas comunicativas y el aprendizaje en general.

Este estudio generó resultados que pueden ser de utilidad para los docentes de idiomas, pues contribuye con información que amplía el escaso corpus de investigación sobre el uso de las estrategias comunicativas en el ámbito universitario en Colombia. No obstante, se presentan algunas limitaciones que deben ser mencionadas. Primero, el instrumento de recolección de información OCSI puede ser triangulado con entrevistas y observaciones de la manera en que los individuos aplican las estrategias en el momento de enfrentar una dificultad a nivel oral. Segundo, en el estudio solo se consideraron las variables de sexo y nivel de dominio del idioma. En efecto, los hallazgos no revelan todos los posibles factores que inciden en la selección y frecuencia de uso de las estrategias para la expresión oral utilizadas por un estudiante. Cabe señalar que se considera inconveniente generalizar estos hallazgos a todos los individuos que estudian inglés en diferentes contextos, más aún cuando en la literatura se encuentran resultados contradictorios frente a la frecuencia de uso de las estrategias comunicativas y sus preferencias al momento de resolver las dificultades que surgen al hablar.

Los aportes de este estudio van en dos direcciones. Por un lado, se contribuye a la discusión teórica y se suma evidencia empírica en la controversia sobre las diferencias en el uso de estrategias comunicativas en cuanto al sexo y el nivel de inglés. Además, si bien se han realizado estudios similares, este es uno de los pocos en Hispanoamérica. Por otro parte, los resultados pueden tener implicaciones prácticas en las metodologías de enseñanza de la segunda lengua. Algunos de los hallazgos invitan a considerar la relevancia de elementos psicopedagógicos en el aprendizaje, como la disposición para comunicarse. Esta variable, asociada con la personalidad, deberá analizarse en otras investigaciones.

Finalmente, como líneas futuras de investigación se proponen los siguientes planteamientos: realizar un estudio donde se lleven a cabo diversas tareas y se graben las interacciones de los estudiantes para identificar las estrategias comunicativas que emplean a nivel del habla y de la escucha y la manera y la frecuencia con que las utilizan. Explorar el impacto que podría tener el entrenamiento formal en el uso de las estrategias comunicativas en los estudiantes y su relación con las variables estilos de aprendizaje, la conciencia de estar aprendiendo una lengua, la motivación y la competencia discursiva. 


\section{Agradecimientos}

Expresamos nuestros agradecimientos a los evaluadores por sus valiosos comentarios, y a las personas que acompañaron y trabajaron en el proceso editorial de esta publicación.

\section{Referencias}

Ahmed, S. T. S., y Pawar, S. V. (2018). Communicative competence in English as a foreign language: Its meaning and the pedagogical considerations for its development. The Creative Launcher, 2(4), 301-312. https://www.thecreativelauncher.com/upload/41.\%20Communicative\%20competence\%20in\%20E FL\%20The-\%20Creative\%20Launcher--\%202018..pdf

Al Alawi, R. A. (2016). Communication strategies used by Omani EFL students. Pyrex Journal of English and Literature, 2(1), 1-11. http://www.pyrexjournals.org/pjel

Berenguer-Román, I., Roca-Revilla, M., y Torres-Berenguer, I. (2016). La competencia comunicativa en la enseñanza de idiomas. Dominio de las Ciencias, 2(2), 25-31. http://dominiodelasciencias.com/ojs/index.php/es/index

Bergil, A. S. (2016). The influence of willingness to communicate on overall speaking skills among EFL learners. Procedia - Social and Behavioral Sciences, 232, 177-187. https://doi.org/10.1016/j.sbspro.2016.10.043

Bergmann, J., y Sams, A. (2012). Flip your classroom: Reach every student in every class every day. Teaching Theology \& Religion (1.. ed.). International Society for Technology in Education. https://doi.org/10.1111/teth.12165

Bonilla-Traña, M. (2016). Enseñanza explícita de estrategias comunicativas en el aprendizaje de la habilidad de expresión oral del inglés como L2 en adultos mayores. Universidad de Concepción. http://repositorio.udec.cl/handle/115 $94 / 2098$

Castillo, M. (2013). La construcción de una competencia comunicativa en el aula como medio para la comprensión de las matemáticas. RIDE Revista Iberoamericana para la Investigación y el Desarrollo Educativo, 3(6), 1-11. http:/ /1-11.ride.org.mx/index.php/RIDESECUNDARIO/article/view/425

Chairat, P. (2017). Oral communication strategies used by english major undergraduates during the internship program. En International Conference on Literature, History, Humanities and Interdisciplinary Studies (pp. 49-52). International Centre of Economics, Humanities and Management. http://eares.org/siteadmin/upload /ED0717032.pdf

Consejo de Europa. (2002). Marco común europeo de referencia para las lenguas: aprendizaje, enseñanza, evaluación. h ttp://cvchttp//cvc.cervantes.es/ensenanza/biblioteca_ele/marco/

De Villa, A. (2018). El enfoque de la conciencia lingüística: la reflexión crítica en el aprendizaje y enseñanza de segundas lenguas. Revista Ibero Americana De Educación, 77(2), 163-174. https://doi.org/10.35362/rie7722843

Demir, Y., Mutlu, G., y Şişman, Y. S. (2018). Exploring the oral communication strategies used by Turkish EFL learners: A mixed methods study. International Journal of Instruction, 11(2), 539-554. https://eric.ed.gov/?id=EJ11749 27

Djandue, B. I. (2012). Pensar en la lengua de aprendizaje: ¿una utopía, una posibilidad o una realidad? Revista RedELE Revista Electrónica de Didáctica ELE, 24, 1-14. http://www.educacionyfp.gob.es/dam/jcr:3446035c-63fb-483 9-aa6b-4a5aa6082de2/2012-redele-24-06bi-dromb--djandue-pdf.pdf

Farzam, M. (2017). The effect of cognitive and metacognitive strategy training on intermediate Iranian EFL learners' willingness to communicate. International Journal of Applied Linguistics and English Literature, 7(1), 193. http s://doi.org/10.7575/aiac.ijalel.v.7n.1p.193

García, O. (2009). Multilingual language awareness and teacher education. En N. Cenoz \& J. Hornberger (Eds.), Encyclopedia of language and education (Vol.6, pp. 385-400). https://doi.org/10.1007/springerreference_60072

Harmer, J. (2010). The practice of english language teaching (4.. ed.). Pearson Longman. 
Jindathai, S. (2017). Communication strategies of Engineering students at a private university institute in Bangkok in the academic year of 2015. The New English Teacher, 11(1), 197-216. http://www.assumptionjournal.au.edu/i ndex.php/newEnglishTeacher/article/view/2448/1759

Keim, L. C., y Delgar, G. F. (2019). Estrategias comunicativas y tareas de interacción oral en L3 presenciales y en línea. RIED. Revista Iberoamericana de Educación a Distancia, 22(2), 225-224. https://doi.org/10.5944/ried.22.2.2 2868

Mei, A., y Nathalang, S. (2010). Use of communication strategies by Chinese EFL learners. Chinese Journal of Applied Linguistics, 33(3), 110-125. http://www.celea.org.cn/teic/91/10091708.pdf

Metcalfe, J., y Noom-Ura, S. (2013). Communication strategy use of high and low proficiency learners of english at a Thai university. Learn Journal, 6(1), 66-87. https://www.tci-thaijo.org/index.php/LEARN/article/view/102 722

Ministerio de Educación Nacional. (2016).Experiencias significativas del programa formadores nativos extranjeros. $\mathrm{h}$ ttp://aprende.colombiaaprende.edu.co/ckfinder/userfiles/files/2_ExpSignificativas del Programa Formadores Nativos.pdf

Moore, P. J. (2018). Task-based language teaching (TBLT). En J. I. Liontas (Ed.), TESOL Encyclopedia of english language teaching (pp. 1-7). Wiley. https://doi.org/10.1002/9781118784235.eelt0175

Nakatani, Y. (2006). Developing an oral communication strategy inventory. Modern Language Journal, 90(2), 151-168. https://doi.org/10.1111/j.1540-4781.2006.00390.x

Nakatani, Y. (2010). Identifying strategies that facilitate EFL learners' oral communication: A class room study using multiple data collection procedures. Modern Language Journal, 94(1), 116-136. https://doi.org/10.1111/j.154 0-4781.2006.00390.x

Ning, H., Dai, X., y Zhang, F. (2010). On gender difference in English language and its Causes. Asian Social Science, 6(2), 126-130. https://doi.org/10.5539/ass.v6n2p126

Ounis, T. (2016). Exploring the use of oral communication strategies by high and low proficiency learners of english\#: Tunisian EFL students as a case study. International Journal of Humanities and Cultural Studies, 3(1), 1077-1098. https://www.ijhcs.com/index.php/ijhcs/article/view/1410

Pandey, M., y Pandey, P. (2014). Better English for Better Employment Opportunities. International Journal of Multidisciplinay Approach and Studies, I(4), 93-100. https://www.researchgate.net/publication/264788119_B etter_English_for_Better_Employment_Opportunities

Salazar-Obeso, W. (2015). Exploring communicative competence development in an EFLT classroom at cursos libres. Zona Próxima, 23, 88-103. http://www.scielo.org.co/pdf/zop/n23/n23a07.pdf

Shirkhani, S., Meigouni, M. M., y Alsadat, E. (2020). Oral communication strategies used by Iranian EFL learners and their relationship with the learners' self-efficacy beliefs and anxiety level. Research in English Language Pedagogy, 8(1), 1-20. https://doi.org/10.30486/relp.2019.669075

Signoret, A. (2003). Bilingüismo y cognición: ¿cuándo iniciar el bilingüismo en el aula? Perfiles Educativos, 15(102), 6-21. http://www.scielo.org.mx/pdf/peredu/v25n102/v25n102a2.pdf

Tuyen, L. V., An, H. T., y Hong, T. K. (2020). Strategies used by undergraduate english-majored students in oral communication. VNU Journal of Foreign Studies, 36(1), 156-179. https://doi.org/10.25073/2525-2445/vnuf s.4506

Wood, D. (2016). Willingness to communicate and second language speech fluency: An idiodynamic investigation. System, 60, 11-28. https://doi.org/10.1016/j.system.2016.05.003

Yaman, Ş., y Özcan, M. (2015). Oral communication strategies used by Turkish students learning English as a foreign language. En M. Pawlak y E.Waniek-Klimczak (Eds.), Issues in teaching, learning and testing speaking in a second language (pp. 143-158). Springer-Verlag.

Yusparizal, Y., Irawati, E., y Anugerahwati, M. (2018). Communication strategies used by ELT students across genders. Jurnal Pendidikan Humaniora, 6(1), 24-47. http://journal.um.ac.id/index.php/jph/article/view/11093/5349 


\section{Notas}

* Artículo de investigación científica. Este artículo se deriva del desarrollo de la tesis doctoral "Estrategias comunicativas y disposición para comunicarse en estudiantes universitarios con nivel lingüístico A2 y B1", realizada en la Universidad Cuauhtémoc por el primer autor del artículo, el cual está enmarcado dentro del proyecto de investigación "Estrategias comunicativas para enfrentar los problemas que surgen en la comunicación oral en encuentros con angloparlantes en el Tecnológico de Antioquia, Institución Universitaria”.

\section{Licencia Creative Commons CC BY 4.0}

Para citar este artículo: Gómez, J. F., Restrepo, J. E., y Parra Fernández, E. (2020). Estrategias comunicativas para enfrentar las dificultades al hablar en inglés en una universidad pública de Colombia. Signo y Pensamiento, 39. https://doi.org/Javeriana.syp39.eced 